\title{
Analysis of Comparison of Fluid Jet Impingement Heat Transfer on Flat Plate Using CFD
}

\author{
K. Siva Satya Mohan ${ }^{1}$ and Dr. S. K. Bhatti ${ }^{2}$ \\ ${ }^{1}$ Asst Professor, Department of Mechanical Engineering, GRIET, Bachupally, \\ Hyderabad-500090 \\ ${ }^{2}$ Professor, College of Engineering, Andhra University, Visakhapatnam, India \\ ${ }^{1}$ kunapulisiva46@gmail.com, ${ }^{2}$ sukhvinder_bhatti@yahoo.com
}

\begin{abstract}
A numerical simulation was performed to study the heat transfer performance of a hot fluid in a confined impinging jet on a flat heated surface. The tests were realized for the following ranges of the governing parameters: the jet thickness is $2 \mathrm{~m}$ and the distance of horizontal jet to heated surface was set to 1 to $3 \mathrm{~m}$. Three different cases are considered in this analysis. They are $H / D=0.5,1$ and 1.5. Fluids like Acetylene and Acetyl chloride are compared in this analysis. Turbulent models considered for this analysis are Spallart Almaras, $k-\omega$ and $k-\varepsilon$. Out of these three $k$ - $\omega$ model gives more heat transfer characteristics. The plate is considered to be stationery. Horizontal Jet with convergent nozzle is considered and compared in this analysis. Finally we found out that Surface Nusselt number, Surface Heat Transfer coefficient plays vital role for better heat transfer calculation.
\end{abstract}

Keywords: Jet Impingement, Heat transfer, $C F D, N_{w}, h_{w}$

\section{Introduction}

Impinging jets have received considerable attention due to their inherent characteristics of high rates of heat transfer besides having simple geometry. Various industrial processes involving high heat transfer rates apply impinging jets. Heat transfer rates in case of impinging jets are affected by various parameters like Reynolds's number, Nozzle plate spacing radial distance from stagnation point, prandtl number, target plate inclination, confinement of the jet, nozzle geometry, curvature of target plate, roughness of the target plate, low scale turbulence intensity, i.e., turbulence intensity at the nozzle exit. Impinging Jets have been used to transfer heat in diverse applications, which include the drying of paper, the cooling of turbine blades and the cooling of a grinding process. Jet Impingement flows can be found in the cooling of hotmetal, plastic, glass sheets, electronics, drying paper, fabric and other applications. Jet Impingement is one of the most efficient solutions of cooling hot objects in Industrial processes as it produces a very high heat transfer rate of forced convection. Impingement heat transfer in axisymmetric air jet using thermocouples to measure the temperature with jet positioning is explained in [1]. The development of radially complete results for the liquid film heat transfer with uniform heat flux is explained by [2].The time averaged and temporal measurements of the heat transfer and fluid flow of an impinging air jet is explained in [3]. Numerical study of multiple circular air jet vertically impinging on a flat plate is explained in [4]. Experimental and theoretical studies of the nanofluid thermal conductivity and heat transfer enhancement is explained by [5]. 


\section{Problem Description}

In the present analysis CFD (Gambit and Fluent 6.3.26) software is used. Gambit is used to design the model of the object and Boundary conditions. I took Rectangular jet for my analysis. We draw all the edges of Jet in gambit using edge option by giving $\mathrm{x}$ and $\mathrm{y}$ coordinates. At the end of jet we keep nozzle for free flow of fluid. I have taken convergent nozzle for my analysis. Draw the rectangular plate with $\mathrm{H} / \mathrm{D}=0.5,1$ and 2.Then we take face option for jet and plate. After that we mesh the edges and faces separately. The purpose of meshing is for free flow of fluid through jet to plate. Then we save the files with an extension of .msh. Figures 1-6 shows the Horizontal Jet impingement heat transfer with convergent nozzle on vertical plate with a gap of 1 to $3 \mathrm{~m}$. After Geometry and meshing we go to FLUENT to review results. We are considered three turbulent models with upwind scheme in FLUENT. They are Spallart-Almaras, k- $\omega$ and $\mathrm{k}-\varepsilon$. The wall material is taken to be copper. The wall properties taken to be fixed. They are, $\mathrm{h}=50 \mathrm{~W} / \mathrm{m} 2 \mathrm{~K}, \mathrm{Q} / \mathrm{V}=15000 \mathrm{~W} / \mathrm{m}^{3}, \mathrm{Q} / \mathrm{A}=15000 \mathrm{~W} / \mathrm{m}^{2}$ and $\mathrm{T}=300 \mathrm{~K}$

CASE-I: $\mathrm{H} / \mathrm{D}=0.5$

WHERE H = JET TO PLATE DISTANCE, D = JET THICKNESS

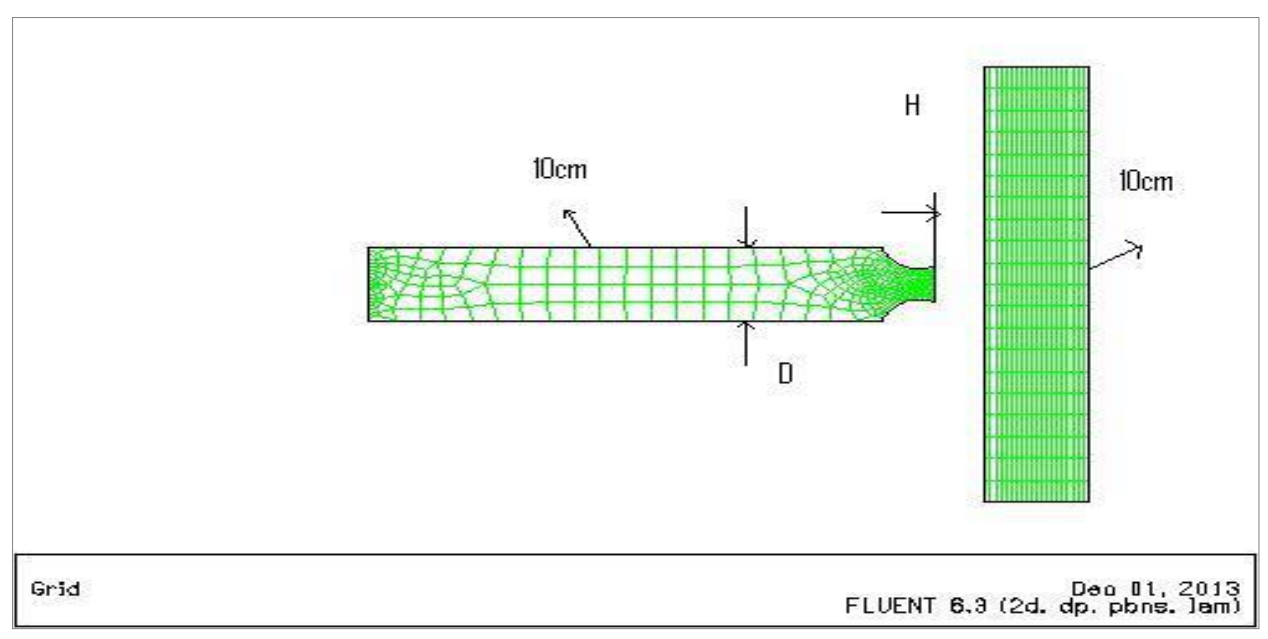

Figure 1. Horizontal Jet with Stationery \& Convergent

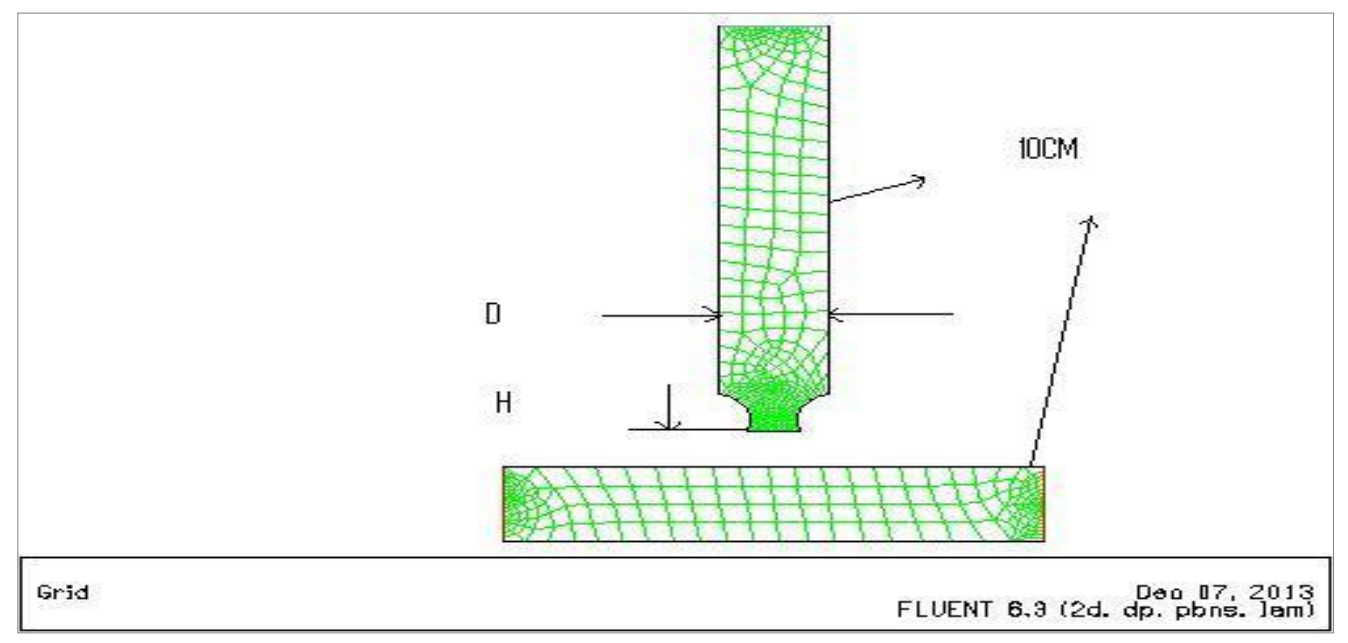

Figure 2. Vertical Jet with Stationery \& Convergent 


\section{CASE-II: $H / D=1$}

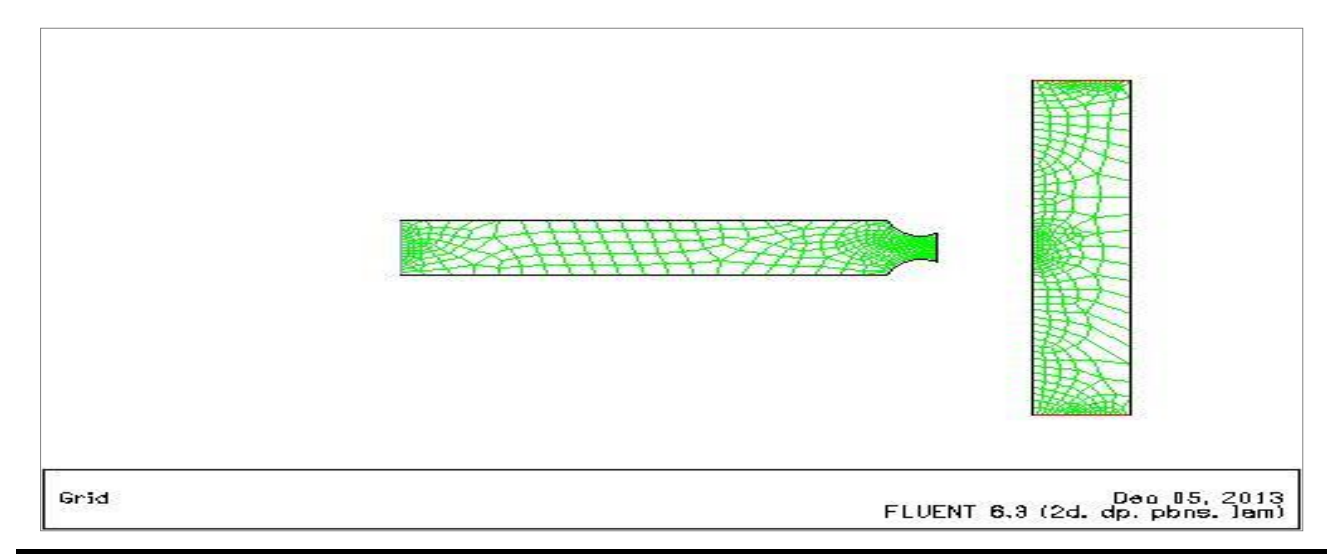

Figure 3. Horizontal Jet with Stationery \& Convergent

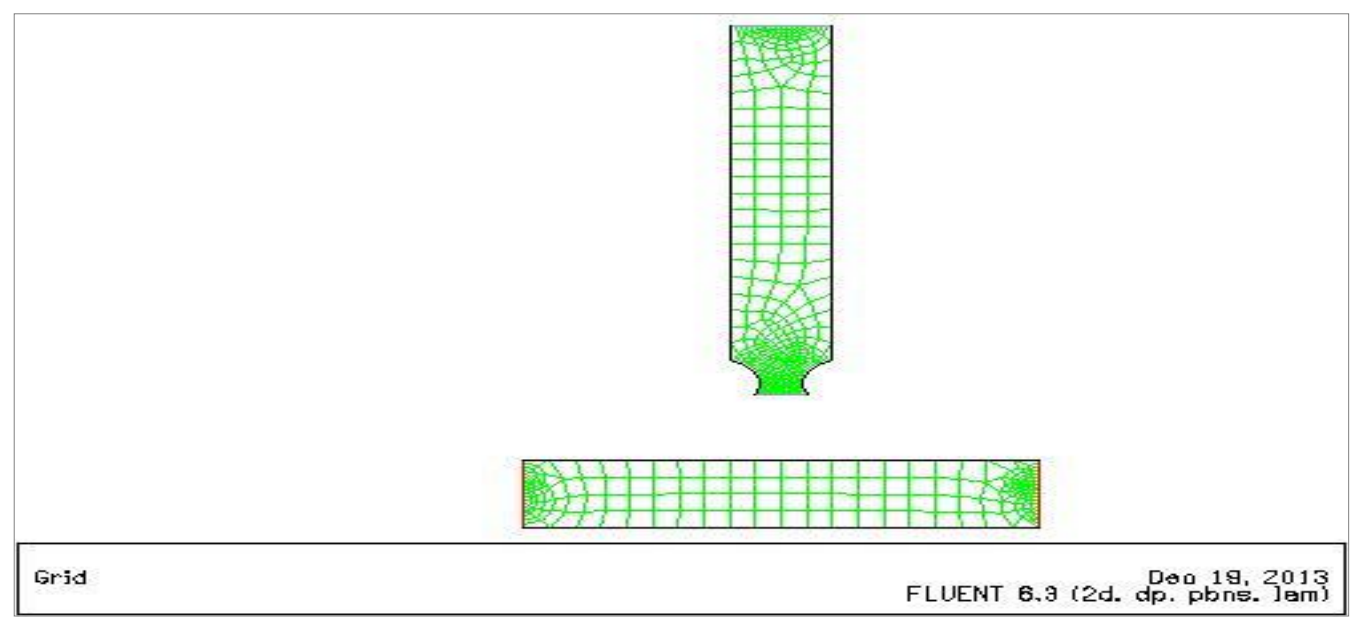

Figure 4. Vertical Jet with Stationery \& Convergent

\section{CASE-III: H/D=1.5}

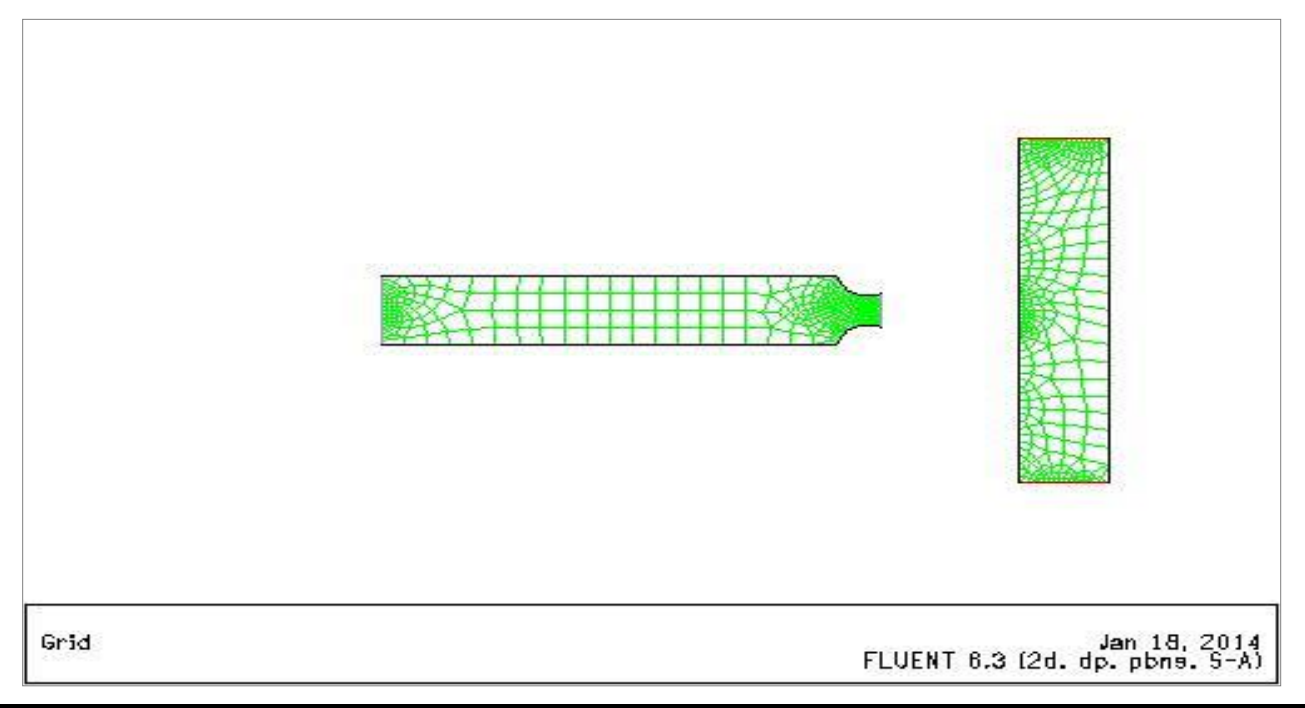

Figure 5. Horizontal Jet With Stationery \& Convergent 


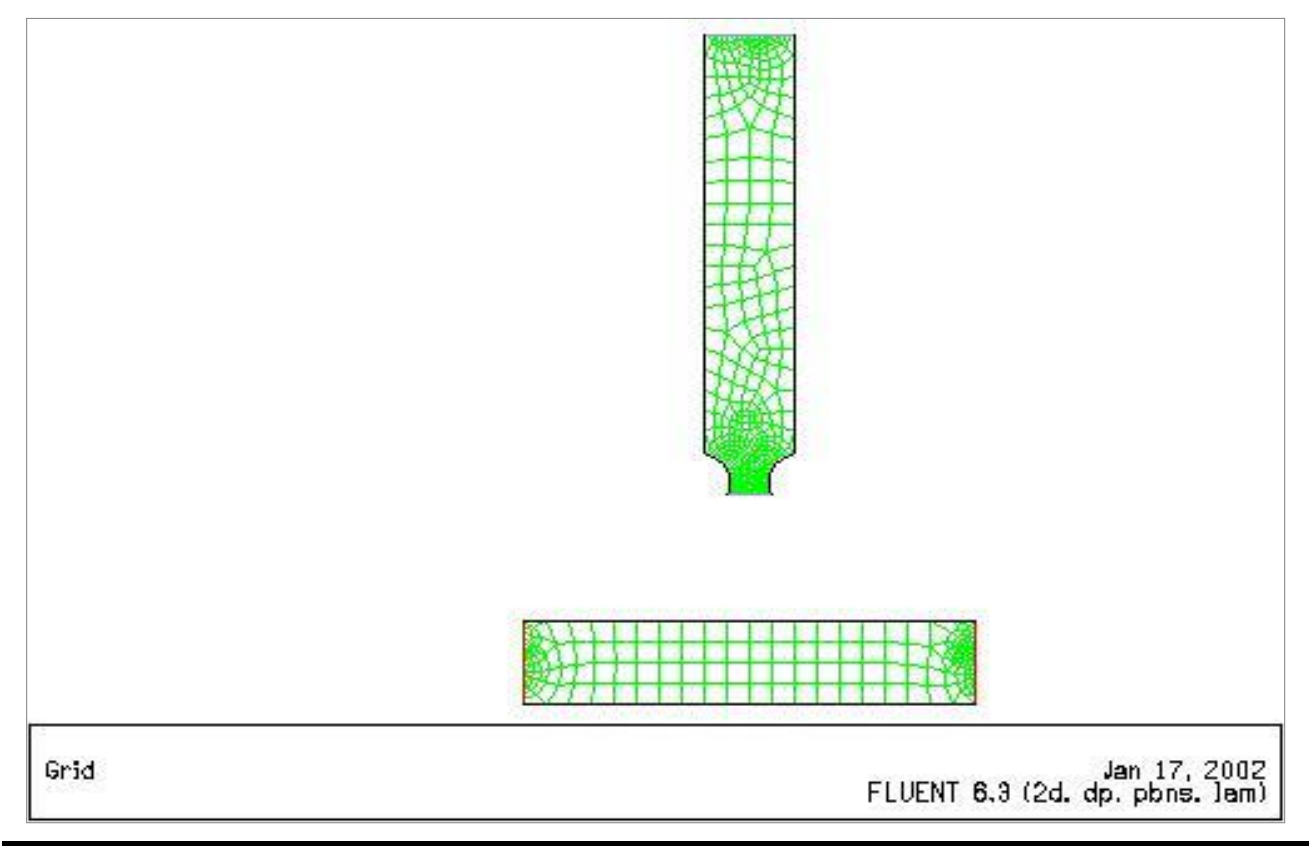

Figure 6. Vertical Jet With Stationery \& Convergent

\section{Boundary Conditions (Table1)}

\begin{tabular}{|c|c|c|c|}
\hline S. No & $\begin{array}{c}\text { Edge } \\
\text { Type }\end{array}$ & $\begin{array}{c}\text { Type In } \\
\text { CFD }\end{array}$ & Value \\
\hline 1 & Inlet & $\begin{array}{c}\text { Pressure } \\
\text { inlet }\end{array}$ & $90000 \mathrm{KPa}$ \\
\hline 2 & Outlet & $\begin{array}{c}\text { Pressure } \\
\text { outlet }\end{array}$ & $75000 \mathrm{KPa}$ \\
\hline 3 & Wall & WALL & ------ \\
\hline
\end{tabular}

4. Thermal Properties of Acetylene \& Acetyl chloride:

Acetylene:

\begin{tabular}{|c|c|}
\hline Property Name & Value \\
\hline Density $\left(\mathrm{kg} / \mathrm{m}^{3}\right)$ & 1.07 \\
\hline Thermal & $\mathbf{0 . 0 2 1 3}$ \\
Conductivity $(\mathrm{W} / \mathrm{mK})$ & \\
\hline Specific heat $(\mathbf{J} / \mathrm{kgK})$ & 2167 \\
\hline Viscosity $(\mathrm{kg} / \mathrm{ms})$ & $1 \times 10^{-5}$ \\
\hline
\end{tabular}

Acetyl Chloride:

\begin{tabular}{|c|c|}
\hline Property Name & Value \\
\hline Density $\left(\mathrm{kg} / \mathrm{m}^{3}\right)$ & 1 \\
\hline $\begin{array}{c}\text { Thermal } \\
\text { Conductivity(W/mK) }\end{array}$ & $\mathbf{0 . 0 4 5 4}$ \\
\hline Specific heat $(\mathrm{J} / \mathrm{kgK})$ & --- \\
\hline Viscosity $(\mathrm{kg} / \mathrm{ms})$ & $\mathbf{1 . 7 2}^{\mathbf{1}} \mathbf{1 0}^{-5}$ \\
\hline
\end{tabular}




\section{CFD ANALYSIS RESULTS:}

A) HORIZONTAL JET WITH H/D $=0.5$ USING ACETYLENE GASCONVERGENT

\begin{tabular}{|c|c|c|c|c|}
\hline SNO & FLUID & METHOD & $\mathbf{H}_{\mathbf{w}}\left(\mathbf{w} / \mathbf{m}^{2} \mathbf{K}\right)$ & $\mathbf{N u}_{\mathbf{w}}$ \\
\hline 1 & ACETYLENE & S-A & 0.14 & 5.8 \\
\hline 2 & ACETYLENE & k- $\varepsilon$ & 9.8 & 390 \\
\hline 3 & ACETYLENE & k- $\boldsymbol{\omega}$ & $\mathbf{1 1 . 1}$ & $\mathbf{4 6 0}$ \\
\hline
\end{tabular}

HORIZONTAL JET WITH H/D $=0.5$ USING ACETYLCHLORIDE GASCONVERGENT

\begin{tabular}{|c|c|c|c|c|}
\hline SNO & FLUID & METHOD & $\mathbf{H}_{\mathbf{w}}\left(\mathbf{w} / \mathbf{m}^{2} \mathbf{K}\right)$ & $\mathbf{N u}_{\mathbf{w}}$ \\
\hline 1 & ACETYLCHLORIDE & S-A & $2.5 \times 10^{-15}$ & $1.2 \times 10^{-13}$ \\
\hline 2 & ACETYLCHLORIDE & k- $\varepsilon$ & $-2.4 \times 10^{-15}$ & $-1 \times 10^{-13}$ \\
\hline 3 & ACETYLCHLORIDE & k- $\boldsymbol{\omega}$ & $\mathbf{7 . 4 \times 1 0 ^ { - 1 5 }}$ & $\mathbf{3} \mathbf{1 0}^{-13}$ \\
\hline
\end{tabular}

B) VERTICAL JET WITH H/D $=0.5$ USING ACETYLENE GAS-CONVERGENT

\begin{tabular}{|c|c|c|c|c|}
\hline SNO & FLUID & METHOD & $\mathbf{H}_{\mathbf{w}}\left(\mathbf{w} / \mathbf{m}^{2} \mathbf{K}\right)$ & $\mathbf{N u}_{\mathbf{w}}$ \\
\hline 1 & ACETYLENE & S-A & 0.23 & 9.5 \\
\hline 2 & ACETYLENE & k- $\varepsilon$ & 0.12 & 4.8 \\
\hline 3 & ACETYLENE & k- $\mathbf{\omega}$ & $\mathbf{1 7}$ & $\mathbf{6 9 0}$ \\
\hline
\end{tabular}

VERTICAL JET WITH H/D $=0.5$ USING ACETYLCHLORIDE GASCONVERGENT

\begin{tabular}{|c|l|c|c|c|}
\hline SNO & FLUID & METHOD & $\mathbf{H}_{\mathbf{w}}\left(\mathbf{w} / \mathbf{m}^{2} \mathbf{K}\right)$ & $\mathbf{N u}_{\mathbf{w}}$ \\
\hline 1 & ACETYLCHLORIDE & S-A & 0.6 & 26 \\
\hline 2 & ACETYLCHLORIDE & k- $\varepsilon$ & 0.6 & 26 \\
\hline 3 & ACETYLCHLORIDE & $\mathbf{k}-\mathbf{\omega}$ & $\mathbf{0 . 6}$ & $\mathbf{2 6}$ \\
\hline
\end{tabular}

C) HORIZONTAL JET WITH H/D $=1$ USING ACETYLENE GASCONVERGENT

\begin{tabular}{|c|c|c|c|c|}
\hline SNO & FLUID & METHOD & $\mathbf{H}_{\mathbf{w}}\left(\mathbf{w} / \mathbf{m}^{2} \mathbf{K}\right)$ & $\mathbf{N u}_{\mathbf{w}}$ \\
\hline 1 & ACETYLENE & S-A & 0.4 & 16 \\
\hline 2 & ACETYLENE & k- $\varepsilon$ & 0.4 & 16 \\
\hline 3 & ACETYLENE & k- $\boldsymbol{\omega}$ & $\mathbf{1 8}$ & $\mathbf{7 5 0}$ \\
\hline
\end{tabular}

HORIZONTAL JET WITH H/D $=1$ USING ACETYLCHLORIDE GASCONVERGENT

\begin{tabular}{|c|c|c|c|c|}
\hline SNO & FLUID & METHOD & $\mathbf{H}_{\mathbf{w}}\left(\mathbf{w} / \mathbf{m}^{2} \mathbf{K}\right)$ & $\mathbf{N u}_{\mathbf{w}}$ \\
\hline 1 & ACETYLCHLORIDE & S-A & $5 \times 10^{-15}$ & $2 \times 10^{-13}$ \\
\hline 2 & ACETYLCHLORIDE & $\mathrm{k}-\varepsilon$ & $3.7 \times 10^{-15}$ & $1.7 \times 10^{-13}$ \\
\hline 3 & ACETYLCHLORIDE & $\mathbf{k}-\boldsymbol{\omega}$ & $\mathbf{3 \times 1 0} \mathbf{1 0}^{-14}$ & $\mathbf{1 . 2 X ~ 1 0}^{-12}$ \\
\hline
\end{tabular}

D) VERTICAL JET WITH H/D = 1 USING ACETYLENE GAS-CONVERGENT

\begin{tabular}{|c|c|c|c|c|}
\hline SNO & FLUID & METHOD & $\mathbf{H}_{\mathbf{w}}\left(\mathbf{w} / \mathbf{m}^{2} \mathbf{K}\right)$ & $\mathbf{N u}_{\mathbf{w}}$ \\
\hline 1 & ACETYLENE & S-A & $-1.3 \times 10^{-15}$ & $-5 \times 10^{-14}$ \\
\hline 2 & ACETYLENE & $\mathrm{k}-\varepsilon$ & $-3.75 \times 10^{-15}$ & $-1.5 \times 10^{-13}$ \\
\hline 3 & ACETYLENE & k- $\boldsymbol{\omega}$ & $\mathbf{- 2 . 4 \times 1 0 ^ { - 1 5 }}$ & $\mathbf{- 1 \times 1 0 ^ { - 1 3 }}$ \\
\hline
\end{tabular}


VERTICAL JET WITH H/D $=1$ USING ACETYLCHLORIDE GASCONVERGENT

\begin{tabular}{|c|l|c|c|c|}
\hline SNO & FLUID & METHOD & $\mathbf{H}_{\mathbf{w}}\left(\mathbf{w} / \mathbf{m}^{2} \mathbf{K}\right)$ & $\mathbf{N u}_{\mathbf{w}}$ \\
\hline 1 & ACETYLCHLORIDE & S-A & $-1.25 \times 10^{-15}$ & $-1.5 \times 10^{-13}$ \\
\hline 2 & ACETYLCHLORIDE & $\mathrm{k}-\varepsilon$ & $-3.6 \times 10^{-15}$ & $-1.5 \times 10^{-13}$ \\
\hline 3 & ACETYLCHLORIDE & $\mathbf{k}-\mathbf{\omega}$ & $\mathbf{- 2 . 4 \times 1 0 ^ { - 1 5 }}$ & $-1 \times 10^{-13}$ \\
\hline
\end{tabular}

E) HORIZONTAL JET WITH H/D $=1.5$ USING ACETYLENE GASCONVERGENT

\begin{tabular}{|c|c|c|c|c|}
\hline SNO & FLUID & METHOD & $\mathbf{H}_{\mathbf{w}}\left(\mathbf{w} / \mathbf{m}^{2} \mathbf{K}\right)$ & $\mathbf{N u}_{\mathbf{w}}$ \\
\hline 1 & ACETYLENE & $\mathrm{S}-\mathrm{A}$ & 0.4 & 16.5 \\
\hline 2 & ACETYLENE & $\mathrm{k}-\varepsilon$ & 0.4 & 16.5 \\
\hline 3 & ACETYLENE & k- $\boldsymbol{\omega}$ & $\mathbf{1 9 . 5}$ & $\mathbf{8 0 0}$ \\
\hline
\end{tabular}

HORIZONTAL JET WITH H/D $=1.5$ USING ACETYLCHLORIDE GASCONVERGENT

\begin{tabular}{|c|c|c|c|c|}
\hline SNO & FLUID & METHOD & $\mathbf{H}_{\mathbf{w}}\left(\mathbf{w} / \mathbf{m}^{2} \mathbf{K}\right)$ & $\mathbf{N u}_{\mathbf{w}}$ \\
\hline 1 & ACETYLCHLORIDE & S-A & $2.5 \times 10^{-15}$ & $1 \times 10^{-13}$ \\
\hline 2 & ACETYLCHLORIDE & k- $\varepsilon$ & $1.5 \times 10^{-15}$ & $5 \times 10^{-14}$ \\
\hline 3 & ACETYLCHLORIDE & k- $\mathbf{\omega}$ & $\mathbf{1 9}$ & $\mathbf{8 0 0}$ \\
\hline
\end{tabular}

F) VERTICAL JET WITH H/D = 1.5 USING ACETYLENE GAS-CONVERGENT

\begin{tabular}{|c|c|c|c|c|}
\hline SNO & FLUID & METHOD & $\mathbf{H}_{\mathbf{w}}\left(\mathbf{w} / \mathbf{m}^{2} \mathbf{K}\right)$ & $\mathbf{N u}_{\mathbf{w}}$ \\
\hline 1 & ACETYLENE & $\mathrm{S}-\mathrm{A}$ & $3.6 \times 10^{-15}$ & $1.5 \times 10^{-13}$ \\
\hline 2 & ACETYLENE & $\mathrm{k}-\varepsilon$ & $3.9 \times 10^{-14}$ & $1.6 \times 10^{-12}$ \\
\hline 3 & ACETYLENE & $\mathbf{k}-\boldsymbol{\omega}$ & $\mathbf{7 . 9} \times \mathbf{1 0}^{-14}$ & $\mathbf{3 . 2 \times 1 0 ^ { - 1 2 }}$ \\
\hline
\end{tabular}

VERTICAL JET WITH H/D $=1.5$ USING ACETYLCHLORIDE GASCONVERGENT

\begin{tabular}{|c|l|c|c|c|}
\hline SNO & FLUID & METHOD & $\mathbf{H}_{\mathbf{w}}\left(\mathbf{w} / \mathbf{m}^{2} \mathbf{K}\right)$ & $\mathbf{N u}_{\mathbf{w}}$ \\
\hline 1 & ACETYLCHLORIDE & S-A & $3.6 \times 10^{-15}$ & $1.5 \times 10^{-13}$ \\
\hline 2 & ACETYLCHLORIDE & $\mathrm{k}-\varepsilon$ & $2.6 \times 10^{-15}$ & $1 \times 10^{-13}$ \\
\hline 3 & $\underline{\text { ACETYLCHLORIDE }}$ & $\mathbf{k}-\mathbf{\omega}$ & $\mathbf{7 . 9} \times \mathbf{1 0}^{-14}$ & $\mathbf{3 . 2 \times 1 0 ^ { - 1 2 }}$ \\
\hline
\end{tabular}

\section{Conclusions/Recommendations}

1. When $H / D=0.5$, Acetylene gas has more values of surface heat transfer coefficient and Nusselt number compared with Acetyl chloride at k- $\omega$ turbulent model.

2. When $H / D=1$, Acetylene gas has more values of surface heat transfer coefficient and Nusselt number compared with Acetyl chloride at k- $\omega$ turbulent model.

3. When $\mathrm{H} / \mathrm{D}=2$, Acetylene gas has more values of surface heat transfer coefficient and Nusselt number compared with Acetyl chloride at k- $\omega$ turbulent model.

4. When $\mathrm{H} / \mathrm{D}=0.5$ Vertical Jet has more heat transfer rate than Horizontal Jet

5. At $\mathrm{H} / \mathrm{D}=1$ and 1.5 Horizontal Jet has more heat transfer rate than Vertical Jet.

6. I conclude that Horizontal jet at $\mathrm{H} / \mathrm{D}=1.5$ is preferable compare to $\mathrm{H} / \mathrm{D}=0.5$ and 1 due to more transfer rate.

7. Also Acetylene gas is preferred than Acetyl chloride for better heat transfer rate. 
8. $\mathrm{k}-\omega$ turbulent model is the best model used for better heat transfer calculation.

9. I also recommend as the distance between Jet and plate increases we can get more transfer rate

10. Finally I recommend Horizontal Jet is mostly suitable in Industrial Application than Vertical Jet for more heat transfer rate.

\section{References}

[1] M. Rahimi, I. Owen and J. Mistry, "Impingement heat transfer in an under-expanded axisymmetric air jet", Department of Engineering, The University of Liverpool, Liverpool L69 3GH, UK published in IJHMT, (2002).

[2] X. Liu, J. H. Lienhard V and J. S. Lombara, "Convective heat transfer by Impingement of Circular Liquid Jets", Department of Mechanical Engineering, Massachusetts Institute of Technology, Cambridge, MA 02139.

[3] T. S. O'Donovan, D. B. Murray, "Effect of vortices on jet impingement Heat Transfer", Department of Mechanical \& Manufacturing Engineering, University of Dublin, Trinity College Dublin, Ireland.

[4] N. K. Chougule, G. V. Parishwad, P. R. Gore, S. Pagins, S. N. Sapali, "CFD Analysis of Multi jet Air Impingement on Flat plate".

[5] T. Kavitha, A. Rajendran, A. Durairajan and A. Shanmugam, "Heat Transfer Enhancement using Nanofluids and innovative methods an overview", Department of Mechanical Engineering, SAMS college of Engineering and Technology, Chennai, Tamilnadu, India. 
International Journal of Advanced Science and Technology Vol.94 (2016) 